\title{
Time Scale Analysis of Precipitation Based on EMD Method in Xilin Gol
}

\author{
Wenjing Suo ${ }^{1}$, Guodong $\mathrm{Wu}^{2}$ and Heru $\mathrm{Xue}^{\mathrm{i}^{*}}$ \\ ${ }^{1}$ College of Computer and Information Engineering,Inner Mongolia Agricultural University,Hohhot, Inner Mongolia,010018,China \\ ${ }^{2}$ College of Computer and Information Engineering,Inner Mongolia Agricultural University,Hohhot, Inner Mongolia,010018,China
}

\begin{abstract}
Xilin Gol is located in the north-central part of Inner Mongolia, and its western plains are extremely scarce areas of water resources. The analysis of the precipitation in Xilin Gol is of great significance to the economic development of local animal husbandry. Time series analysis and prediction have important practical application value, and scientific and accurate prediction results can provide a key guiding role for social activities.Combined with the fast Fourier transform, the empirical modal decomposition method was used to analyze the monthly precipitation data of Xilin Gol from 1961 to 2016. The results show that the time series has a period of 6 and about 22 months.And through the calculation of the contribution rate of the IMF variance, it is found that 12 months is the main cycle of the monthly precipitation of Xilin Gol,The results show that EMD can more accurately reflect the periodic characteristics of precipitation. The research results have certain practical value for the scientific and rational use of precipitation.
\end{abstract}

\section{Introduction}

Xilin Gol is located in the northwestern part of Inner Mongolia. The economic structure is mainly based on agriculture and animal husbandry, and it is also an important livestock product base of the country. And the western plain area is an area with extreme water resources. Studying the water resources of Xilin Gol has certain effects on its drought prevention and mitigation and sustainable development of animal husbandry. Therefore, the detection and management of water resources is of great significance to its economic development ${ }^{[1]}$.

For time series scale analysis, related studies have been carried out. In 2008, Li Xingyan ${ }^{[2]}$ used the EMD method to analyze the time series of the natural runoff of the Weihe River, and revealed the trend changes and influencing factors of each component. In 2010, Lu Jingwei ${ }^{[3]}$ selected the Weihe River Basin as the research object to study the variation characteristics of hydrometeorological sequences by using the empirical modal decompositionm method and wavelet analysis method. In 2012, Deng-Fa Lin, Po-Hung Chenliyong ${ }^{[4]}$ used empirical mode decomposition and fast Fourier transform to analyze the oil leakage fault signal of wind power gearbox, and performed FFT on the eigenmode function obtained by EMD decomposition. Find the IMF spectrum to identify the failure of the gearbox. In 2013, Xue Chunfang ${ }^{[5]}$ used ensemble empirical mode decomposition to carry out the multi-scale analysis of the autumn precipitation in the Weihe River Basin in the past

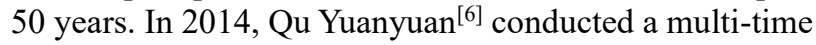
scale analysis of precipitation in Harbin. The results show that twelve months is the main period of precipitation fluctuation. In 2018, $\mathrm{Du} \mathrm{Yi}{ }^{[7]}$ used wavelet analysis to analyze the components of EMD decomposition to obtain the periodic characteristics. In 2018, Fang Aihua ${ }^{[8]}$ used EMD to analyze the temperature of Hui'an County on multiple time scales to obtain the characteristics of temperature cycle transformation.

\section{Theoretical Basis}

\subsection{EMD}

EMD is a method to deal with non-stationary and nonlinear data sequences. It is a smoothing process of a signal, which decomposes the fluctuations of different scales in the signal step by step to produce a series of IMF components containing different time scale feature information of the original signal, that is, the intrinsic mode function.

Steps of the EMD algorithm:

- $\quad$ Determine all local maximum and minimum in $x(t)$;

- The maximum and minimum values are respectively connected by the envelope, and the cubic spline interpolation is usually used, which are respectively set to $\mathrm{e}_{\mathrm{a}}(\mathrm{t})$ and $\mathrm{e}_{\mathrm{b}}(\mathrm{t})$;

- Calculate the mean of the upper and lower envelopes, set to $\mathrm{m}_{1}(\mathrm{t})$,

$$
\mathrm{m}_{1}(t)=\frac{e_{a}(t)+e_{b}(t)}{2}
$$

- make $h_{1}^{1}(t)=x(\mathrm{t})-m_{1}(t)$, check if $h_{1}^{1}(t)$ satisfies the two definitions of IMF. If $h_{1}^{1}(t)$ satisfies 
the two definitions of IMF, it means that we have decomposed an IMF; Otherwise, we replace $x(t)$ with $h_{1}{ }^{1}(t)$ and repeat the process to get an IMF. Then $\mathrm{c} 1(\mathrm{t})=\mathrm{imf}_{1}(\mathrm{t})$;

- $\quad$ By subtracting $\operatorname{imf}_{1}(\mathrm{t})$ from the original signal $\mathrm{x}(\mathrm{t})$, a new signal $r_{1}(t)$ with high frequency components is obtained. The process is repeated for $r_{1}(t)$ and the second IMF component is obtained, which is repeated until the stopping condition is satisfied.

\subsection{Endpoint effect}

In the process of "screening" of IMF, the cubic spline function which constitutes the upper and lower envelopes will diverge at both ends of the data sequence because the end of the sequence may be considered as the extreme value, and this phenomenon will affect the whole sequence inward, which will distort the result ${ }^{[8]}$. In this paper, the method used to deal with the endpoint effect is the polynomial fitting extension method. In the method of obtaining the IMF component of the original data sequence, it first compares the size of the leftmost and rightmost data with the neighboring extreme point to determine whether the endpoint is maximal or minimum. The fitting polynomial is obtained by the three extreme points at the left and right ends of the extreme value sequence (all extreme points are selected when there are not enough three extreme points). Then, by fitting polynomials, we can obtain two approximations of the endpoint sequence and add them to both ends of the data sequence. Finally,find the upper and lower envelopes of the whole data sequence.

\section{Experiment}

\subsection{Data used in the experiment}

The precipitation data of 672 months in Xilingol from 1961 to 2016 were selected as samples. The data are derived from the precipitation grid data of National Meteorological data, and the final experimental data are obtained according to the longitude and latitude of Xilingol.

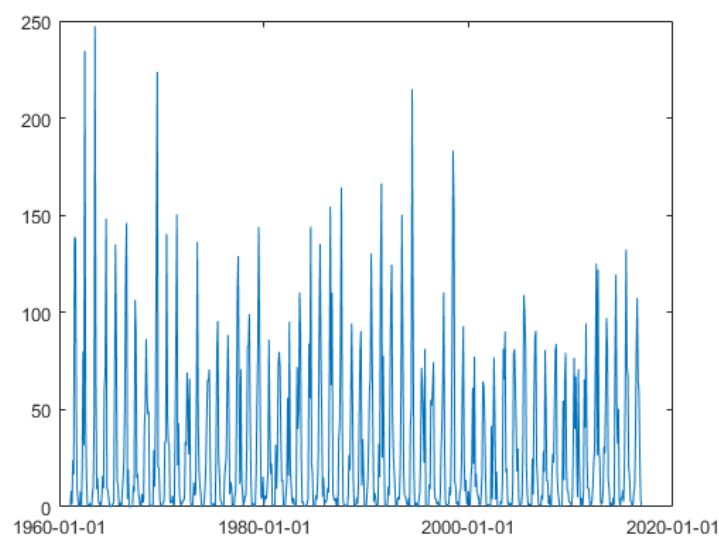

Figure 1. Time series diagram of precipitation.

\subsection{Experimental results and analysis}

The intrinsic mode functions diagram of the original time series decomposed by EMD is shown in Fig.2.The decomposition result consists of seven eigenmode functions and one trend term.

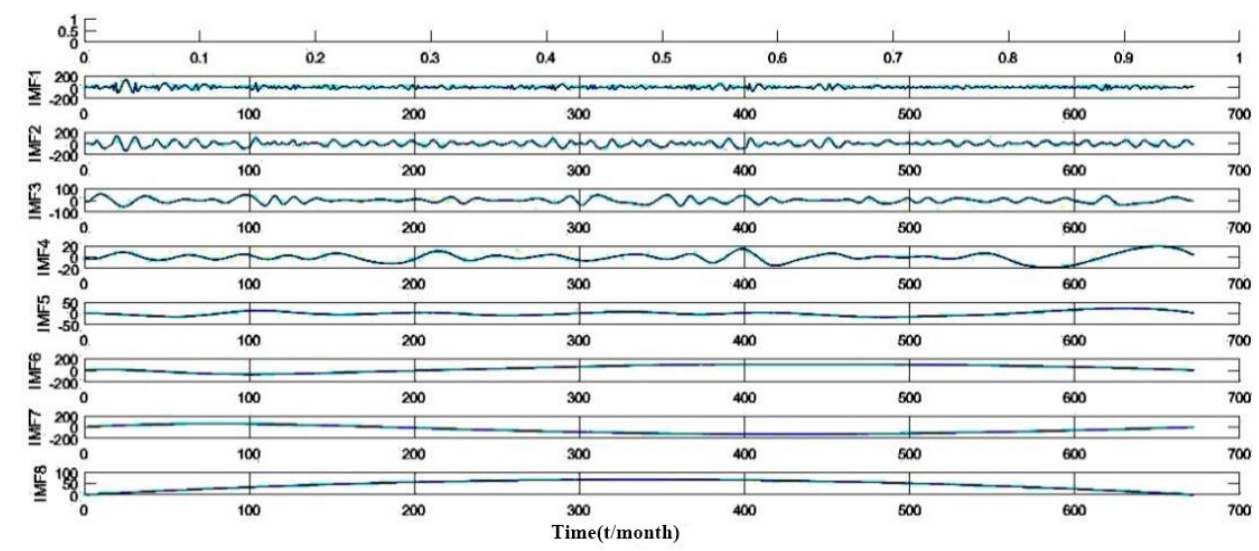

Figure 2. components diagram of IMFs.

The average period of the eigenmode function is obtained by fast fourier transform.It can be seen from the results that $\mathrm{IMF}_{1}$ has the largest amplitude, the highest frequency and the shortest wavelength, showing a short period of six months (the average period is $0.5 \mathrm{a}$ ). Compared with $\mathrm{IMF}_{1}, \mathrm{IMF}_{2}$ has a reduced fluctuation period with an average period of twelve months, which is consistent with the characteristics of the precipitation period.For $\mathrm{IMF}_{3}$, its amplitude is reduced, the wavelength is increased, and it has an average period of about 22 months. The changes of the following components are not significant in the observation period, with an average period of 135 months, 224 months and 672 months respectively. The variance contribution rate of each component and its correlation coefficient with the original sequence are calculated. Tab.1 shows the index of each IMF.

The correlation coefficients between the first three components and the original sequence are more than $10 \%$, which has a great influence on the sequence. And the 
cumulative variance contribution rate of the first three components reaches more than $75 \%$, and these three components are the main contributions of the precipitation sequence change. Since the monthly precipitation data is selected, the analysis of the trend item shows that the monthly data shows a trend of increasing first and then decreasing.

Table 1. Indicators of IMF components.

\begin{tabular}{llllllll}
\hline Component & $\mathrm{IMF}_{1}$ & $\mathrm{IMF}_{2}$ & $\mathrm{IMF}_{3}$ & $\mathrm{IMF}_{4}$ & $\mathrm{IMF}_{5}$ & $\mathrm{IMF}_{6}$ & $\mathrm{IMF}_{7}$ \\
\hline Average Period & 6 & 12 & 21.6774 & 134.4 & 224 & 336 & 672 \\
Variance Contribution Rate (\%) & 19.9317 & 46.9 & 11.5459 & 2.5066 & 2.7199 & 1.9256 & 4.8622 \\
Correlation Coefficient(\%) & 26.17 & 68.62 & 20.98 & 6.03 & 4.95 & 3.34 & -2.39 \\
\hline
\end{tabular}

\section{Conclusions}

The change of the monthly precipitation sequence of Xilin Gol is mainly caused by the periodic oscillation of the first, second and third components, which reflect the cyclical changes of quasi-monthly, quasi-12 months and about 22 months. The characteristics of the latter components are basically the same. The second component contributes a large percentage to the sequence, which is also consistent with the precipitation climate. For the sequence reconstruction, it is recommended to select the first three high-frequency components and trend items, and allocate the weight coefficients according to the correlation or variance contribution rate of each sequence, which may effectively improve the accuracy of the sequence prediction.

\section{Acknowledgments}

This work is supported by national and international scientific and technological cooperation special projects (No.2015DFA00530) and national natural science foundation of China (No.61461041).

\section{References}

1. Zhao Y S,Li R T. Wind resources status and wind farm construction in Xilin Gol League[J].Inner Mongolia Science Technology\&Economy,2012(10):57-58.

2. Li X Y,Ding Z H. EMD Method for Multiple Timescale Analysis on Fluctuation Characteristic of Natural Annual Runoff Time Series of Fen River[J].Water Resources and Power,2008,26(1):3032.

3. Lv J W.Study on the runoff change characteristic and forecast model in jinghe river basin[D]. Northwest A\&amp;F University,2010.

4. Lin D F , Chen P H . Fault Recognition of Wind Turbine Using EMD Analysis and FFT Classification[C].

5. Xue C F,Hou W,Zhao J H. The application of ensemble empirical mode decomposition method in multiscale analysis of region precipitation and its response to the climate change[J]. Acta Phys. Sin,2013,62(10):496-503.

6. Qu Y Y,Qi C Y. Multi-time scale analysis of precipitation in Harbin based on $\operatorname{EMD}[\mathrm{J}]$. Water
Conservancy Science and Technology and Economy,2014.

7. Du Y,Ma R Y,Zhao L Y. An EMD periodic analysis method combining with wavelet analysis[J].Guangxi Water Resources\& Hydropower Engineering,2018(1)

8. Fang A H,Huang D Y,Wang W E. Multi- tim e Scale Analysis of Temperature based on Empirical Mode Decomposition (EMD) Method in Hui' an County[J]. Journal of Agricultural Catastrophology,2018,8(2).

9. Huang N E, Shen Z, Long S R, et al. The empirical mode decomposition and the Hilbert spectrum for nonlinear and non-stationary time series analysis[J]. Proceedings A, 1998, 454(1971):903-995.

10. Yang J W,Jia M P. Study on processing method and analysis of end problem of Hilbert-Huang spectrum[J]. Journal of Vibration Engineering,2006.

11. Xu D,Huang H L. Multi - scale Analysis on Temperature and Precipitation Series in Shenyang During[J]. Journal of Arid Meteorology,2019, 37(02):83-90+97. 